\title{
Primary prostate cancer synchronous with renal cell carcinoma: clinical experience and literature review
}

\author{
ZHONG-YUAN Li, CHENG-CHENG Ying, ZHI-HuA WAN, ZHI-SHUn WANG, GUO-HAO LI, \\ LIN CHEN, YONG-LIAN GUO
}

\begin{abstract}
Department of Urology, The Central Hospital of Wuhan, Tongji Medical College, Huazhong University of Science
\end{abstract} and Technology, Wuhan, People Republic of China

\begin{abstract}
The objective of this study was to report the diagnosis and treatment results of primary prostate adenocarcinoma (PRAD) concurrent in a patient with renal cell carcinoma (RCC), and to review the relative literature. A 62-year-old man was admitted to our hospital with chief complaint of a painless, incidentally found renal mass for one year. RCC was initially found by computed tomography (CT) scan, but prostate cancer was incidentally found by abnormal prostate-specific antigen (PSA) level results. The post-nephrectomy pathology assay reported clear RCC with positive staining of vimentin, cluster of differentiation 10 (CD10), carbonic anhydrase IX (CA-IX), paired box 8 (Pax-8), epithelial membrane antigen (EMA), and Ki67 labeling index (Ki67 LI). Magnetic resonance imaging (MRI) revealed uneven signals in the right peripheral zone of the prostate. Both prostate biopsy and post-prostatectomy pathology examination revealed prostate acinar adenocarcinoma with positive staining of P504S and Ki67 LI. The patient has been in periodic follow-up and has remained in good general condition without any evidence of recurrence to date. To the best of our knowledge, the present report is the only case of systematically described pre- and post-therapy laboratory, pathology, and imaging examination results. Our report together with published studies suggest that increased awareness of synchronous PRAD risk will enable early detection and prompt therapies in patients with RCC.
\end{abstract}

Keywords: renal cell carcinoma, prostate adenocarcinoma, multiple primary malignancies.

\section{a Introduction}

In 2018 , it was estimated that there were 403262 new cases and 175098 new fatalities of renal cancer, representing approximately $2.2 \%$ and $1.8 \%$ of all cancers, respectively [1]. Renal cell carcinoma (RCC), which originates in the kidney cortex, accounts for $80-85 \%$ of all major kidney neoplasms and is one of the 10 most prevalent cancers worldwide [2]. There is evidence that the incidence of this cancer is increasing, although its mortality rates have decreased. Rabbani et al. [3] found that 26.9\% of patients treated for RCC have other antecedent, synchronous, or subsequent primary malignancies including prostate, bladder, and rectal cancers, and non-Hodgkin's lymphoma (NHL) [3]. However, the concurrence of RCC and synchronous prostate cancer $(\mathrm{PCa})$ is still rare.

\section{Aim}

In this study, we present a case of a 62-year-old male, in whom RCC was initially found by a computed tomography (CT) scan, but synchronous prostate adenocarcinoma (PRAD) was suspected due to abnormal prostatespecific antigen (PSA) results. Further imaging and pathology evidence strongly supported our diagnosis of concurrent primary RCC and PRAD. To the best of our knowledge, the present report is the only case of systematically described pre- and post-therapy laboratory, pathology, and imaging examination results. Our study has important implications in management strategies for RCC patients with newly diagnosed PCa.

\section{ㅁ Case presentation}

On April 8, 2019, a 62-year-old man was admitted to our hospital with the chief complaint of a painless, incidentally found renal mass for one year. No kidney percussion and no defects in the chest, stomach and extremities were disclosed by physical examination. Plain CT scan and contrast-enhanced CT of the abdomen revealed a $4.4 \times 3.8 \times 3.9 \mathrm{~cm}$ round mass lesion $(31 \mathrm{HU})$ in the upper pole of the left kidney (Figure 1). The mass was rapidly enhanced after injection of the contrast agent, followed by a quick wash out. No tumor thrombus was observed in bilateral renal veins and inferior vena cava and no swollen retroperitoneal lymph nodes were detected. Renal dynamic imaging showed the glomerular filtration rate of left, right, and total renal were $30.4 \mathrm{~mL} / \mathrm{min}, 36.4 \mathrm{~mL} / \mathrm{min}$ and $66.8 \mathrm{~mL} / \mathrm{min}$, respectively, suggesting a slight reduction in left renal perfusion, left renal function, and total renal function as well as normal right renal perfusion and function. Taken together, a diagnosis of RCC was considered. In the meantime, his laboratory test results were within the normal range, with the exception of elevated total PSA (tPSA) of $6.026 \mathrm{ng} / \mathrm{mL}$, normal free PSA (fPSA) of $0.738 \mathrm{ng} / \mathrm{mL}$, but decreased fPSA $/$ PPSA of $12.2 \%$. The rectal examination showed degree I prostate hyperplasia with a soft surface (no nodules), solid quality, and shallow central sulcus. Further magnetic resonance imaging (MRI) revealed benign prostatic hyperplasia, prostatitis, and uneven signals in the prostate right peripheral zone (Figure 2).

This is an open-access article distributed under the terms of a Creative Commons Attribution-NonCommercial-ShareAlike 4.0 International Public License, which permits unrestricted use, adaptation, distribution and reproduction in any medium, non-commercially, provided the new creations are licensed under identical terms as the original work and the original work is properly cited. 

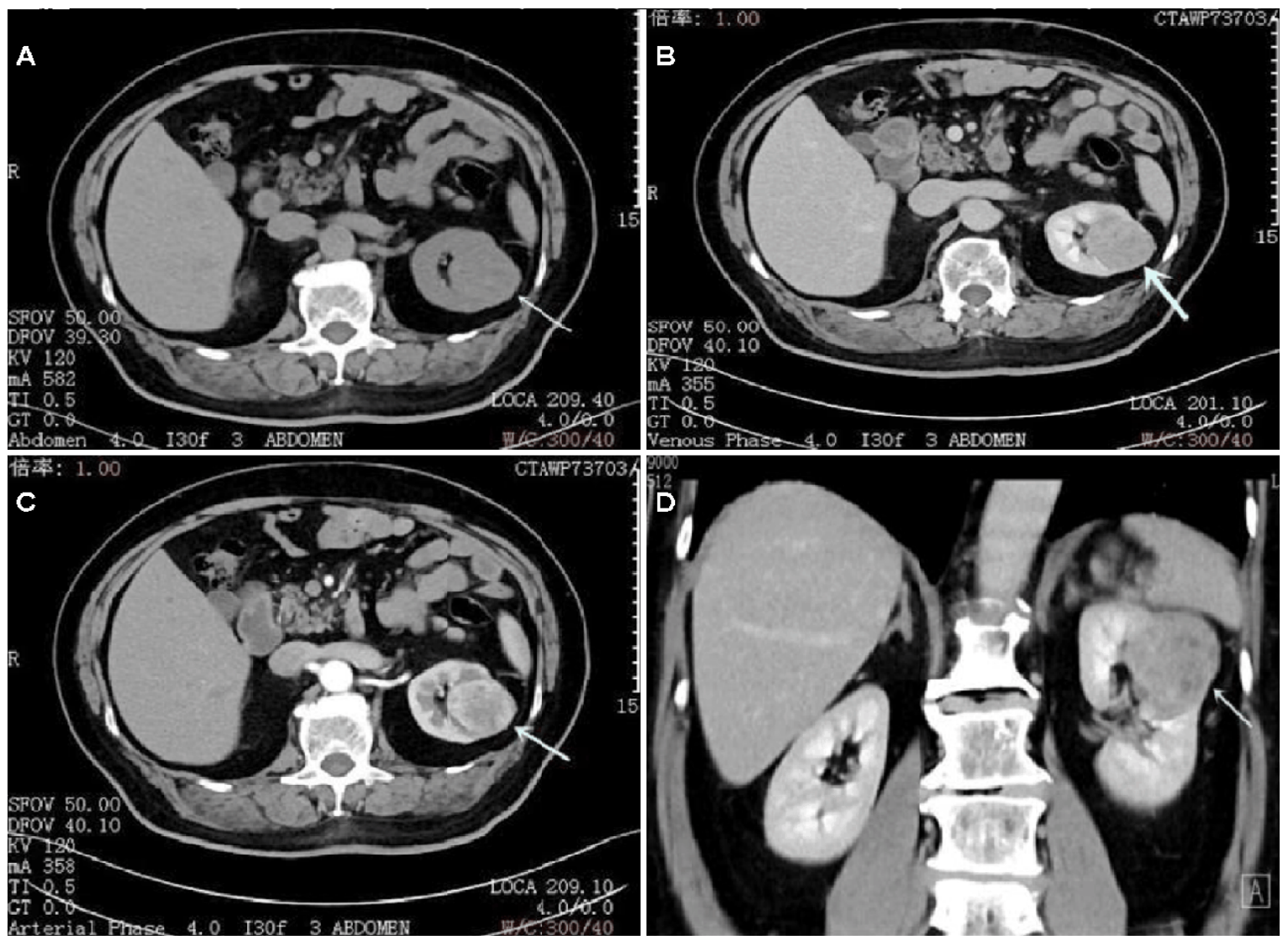

Figure 1 - Preoperative abdominal plain computed tomography (CT) scan (A), venous phase (B), and artery contrastenhanced CT $(C)$ revealed a $4.4 \times 3.8 \times 3.9 \mathrm{~cm}$ round mass lesion $(31 \mathrm{HU})$ in the upper pole of the left kidney. After contrast agent injection, the mass was rapidly enhanced, followed by a quick washing out. No tumor thrombus in the bilateral renal veins and inferior vena cava was observed and no swollen retroperitoneal lymph nodes were detected. (D) The three-dimensional reconstruction of the coronal section of venous phase enhanced CT suggests a solid tumor in the upper pole of the left kidney.

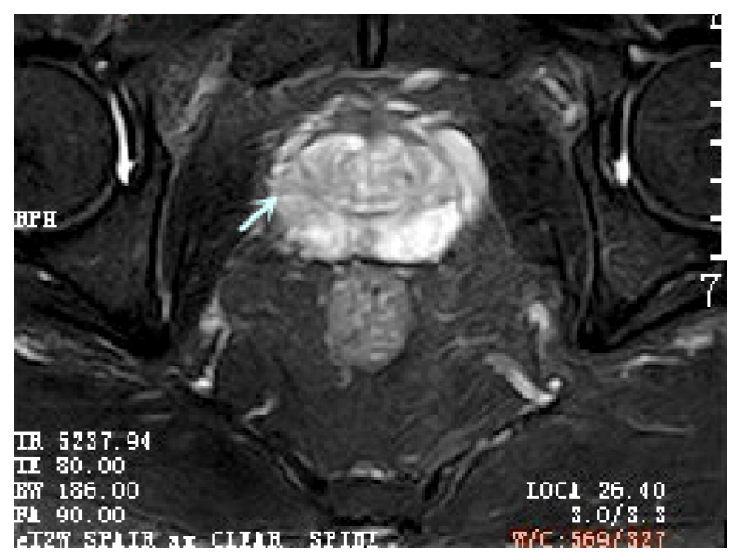

Figure 2 - Prostate magnetic resonance imaging (MRI) revealed benign prostatic hyperplasia, prostatitis, and uneven signals in the prostate right peripheral zone (arrow).

Transrectal prostate biopsy was conducted under color ultrasound guidance and six-needle aspiration was gathered respectively from the left and right lobes. Chronic inflammation and focal atypical small acinar hyperplasia were noted in the histopathological (HP) examination of the left prostate tissue (Figure 3A). As shown in Figure 3B, HP examination of the right prostate tissue suggested PRAD [Gleason grade: $3+3=6$, International Society of Urological Pathologists (ISUP) group 1]. Immunohistochemistry (IHC) analysis showed negative staining of cytokeratin (CK) 5/6, p63, and keratin 903 (K903) and positive staining of P504S (Figure 4A) and Ki67 (1\%) (Figure 4B) interspersed with low proliferation. Taken together, the patient was diagnosed with low-risk localized PRAD (T1cN0M0), which was momentarily and actively controlled. Therefore, laparoscopic partial nephrectomy of the left kidney tumor was first conducted under general anesthesia. Postoperative HP examination reported clear RCC ( $4 \times 4 \mathrm{~cm}$ in size, Fuhrman nuclear grade II; Figure 5A). IHC showed negative staining for cluster of differentiation (CD) 117 and CK7, weak positive staining of RCC and P504S, and positive staining of vimentin, CD10, carbonic anhydrase IX (CA-IX), paired box 8 (Pax-8), epithelial membrane antigen (EMA), and Ki67 labeling index (Ki67 LI) $(3 \%)$ (Figure 6, A-H). The patient recovered and was discharged five days after surgery.

The patient was readmitted for confirmed PRAD for more than two months, returning to the hospital for surgery on August 7, 2019. There was no solid organ and bone metastasis as identified by bone scintigraphy. The detected concentrations of tPSA and IPSA were $6.317 \mathrm{ng} / \mathrm{mL}$ and $0.625 \mathrm{ng} / \mathrm{mL}$, respectively, resulting in decreased fPSA/tPSA (9.9\%). The plain and contrast-enhanced CT scan indicated that there was no tumor recurrence in the left kidney (Figure 5B). Under general anesthesia, laparoscopic radical prostatectomy was performed. The pelvic drainage tube was removed four days after the operation and the catheter was removed four weeks post- 
operation. Urination was smooth, with a peak urinary flow rate of $15 \mathrm{~mL} / \mathrm{s}$, and no urinary incontinence was observed. The patient was discharged five days after surgery. Postoperative HP examination confirmed the diagnosis of prostate acinar adenocarcinoma (Gleason score: $3+4=7$, ISUP group 2) with local visible neurological invasion (Figure 7A). IHC showed negative staining of CK5/6, p63, and K903, and positive staining of P504S and Ki67 LI

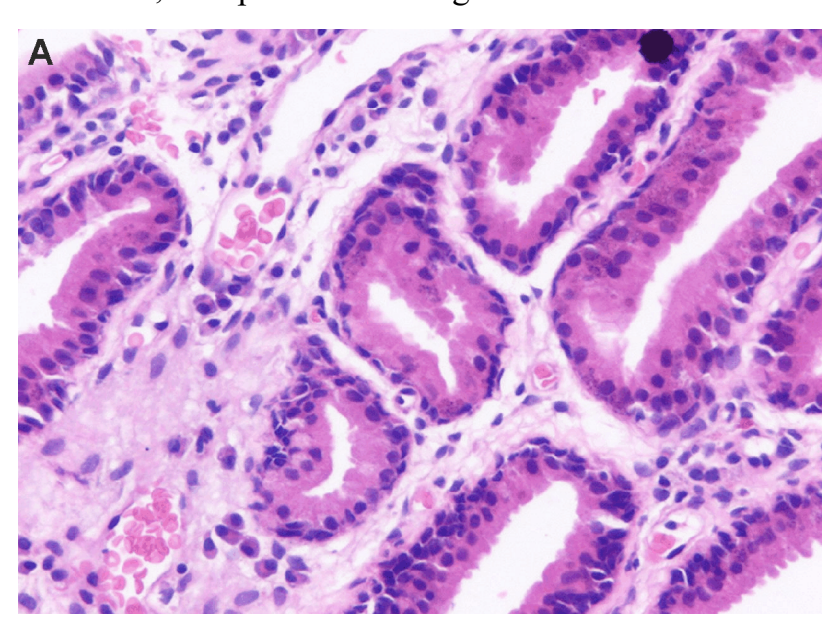

( $1 \%)$. No tumor tissue was observed in the cutting edge of the prostate tip, bilateral vas deferens, bilateral seminal vesicles, or prostate bottom margin. The laboratory test results also found that the concentrations of tPSA, fPSA, and fPSA/tPSA decreased to $0.017 \mathrm{ng} / \mathrm{mL}, 0.001 \mathrm{ng} / \mathrm{mL}$ and $5.9 \%$, respectively. At two months after radical prostatectomy, the three-dimensional reconstruction of the urinary plain CT scan showed no tumor recurrence (Figure 7B).

Figure 3 - In the HP examination of transrectal prostate biopsy for the left prostate tissue, chronic inflammation, and focal atypical small acinar hyperplasia was observed (A). For the right prostate tissue, HP examination indicated prostate adenocarcinoma (Gleason grade: 3+3=6, ISUP group 1) (B). HE staining: (A and B) 200×. HE: Hematoxylin-Eosin; HP: Histopathological; ISUP: International Society of Urological Pathologists.
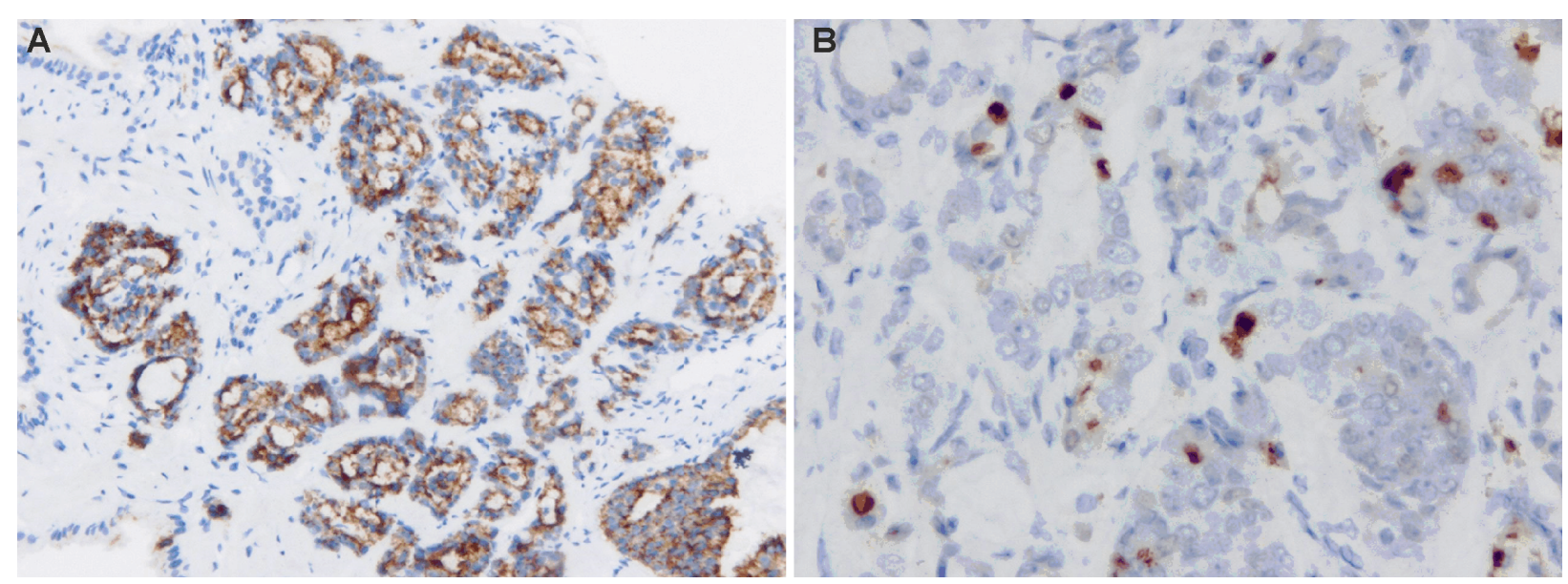

Figure 4 - Immunohistochemistry analysis (200×) of the transrectal biopsy from right prostate showed positive staining of P504S (A) and Ki67 (B).

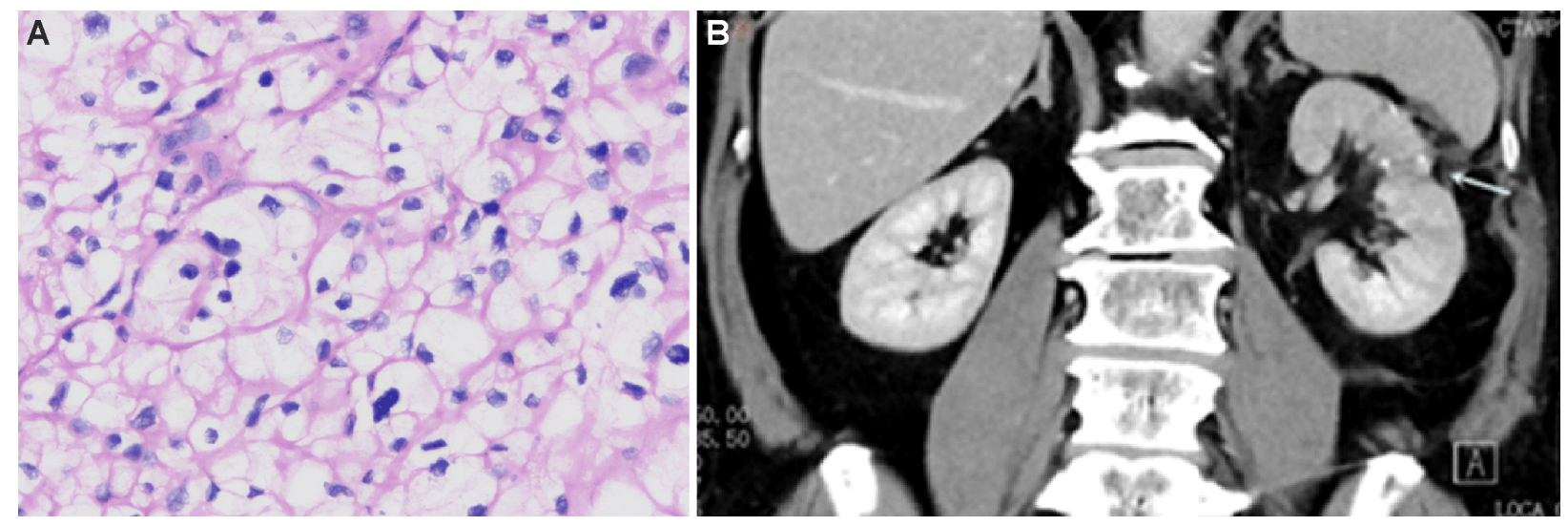

Figure 5 - (A) Postnephrectomy histopathological examination showed clear renal cell carcinoma $(4 \times 4 \mathrm{~cm}$ in size, Fuhrman nuclear grade II) (HE staining, 200×). (B) At three months after nephrectomy, the three-dimensional reconstruction of the coronal section of contrast-enhanced CT indicated no tumor recurrence in the left kidney (arrow). CT: Computed tomography; HE: Hematoxylin-Eosin. 


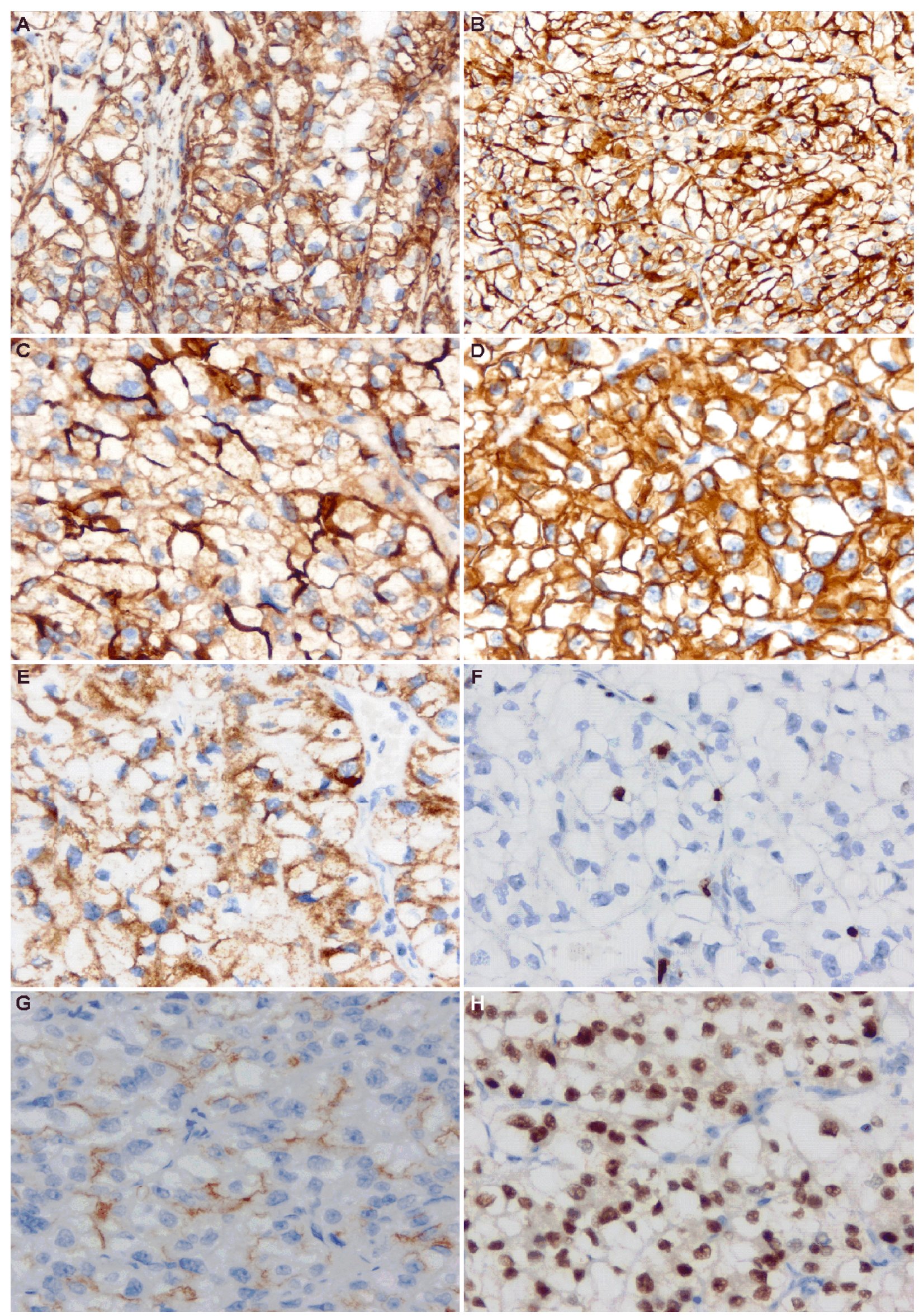

Figure 6 - Immunohistochemistry analysis (200×) of the left kidney tumor after laparoscopic partial nephrectomy showed positive staining of vimentin(A), CD10 (B), EMA (C), CA-IX (D), P504S (E), Ki67 (F), RCC (G), Pax-8 (H). CA-IX: Carbonic anhydrase IX; CD10: Cluster of differentiation 10; EMA: Epithelial membrane antigen; Pax-8: Paired box 8; RCC: Renal cell carcinoma. 


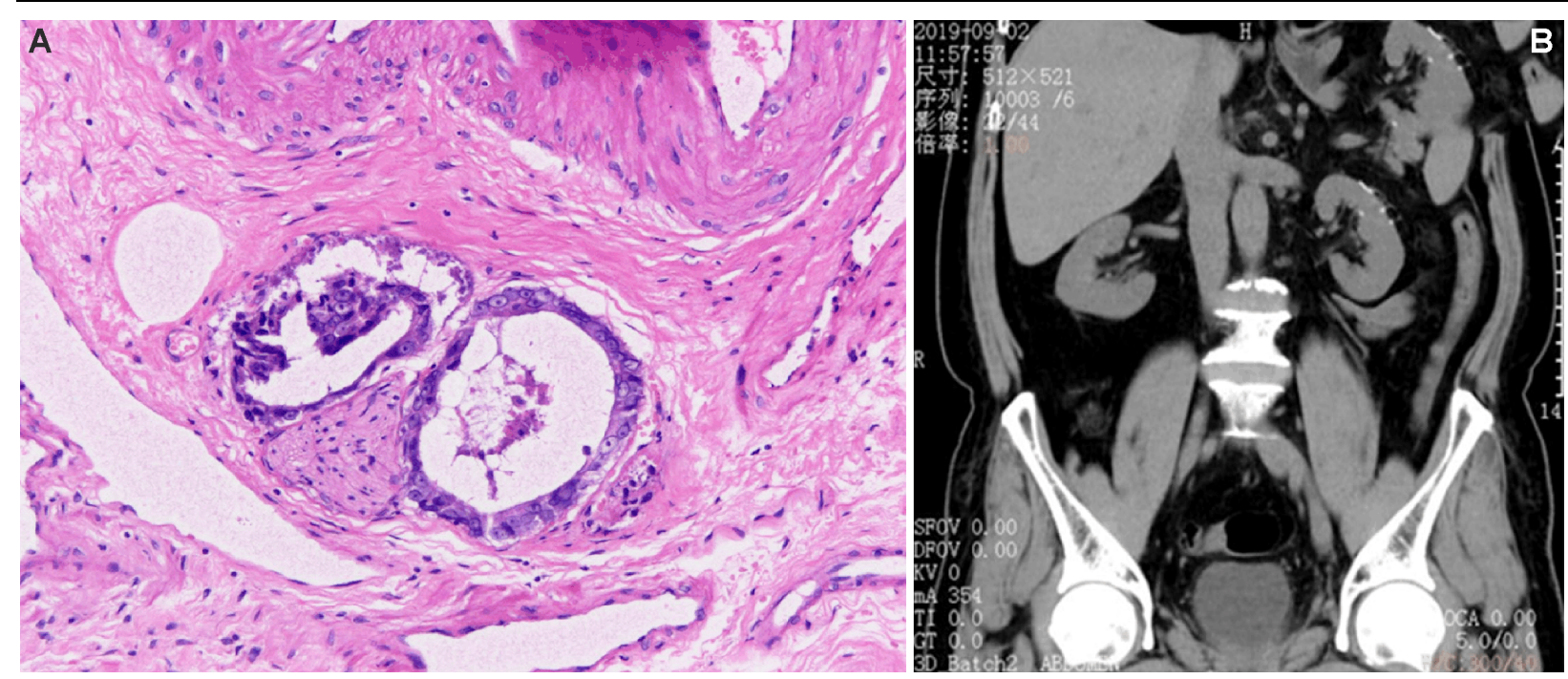

Figure 7 - (A) Postprostatectomy histopathological examination detected prostate acinar adenocarcinoma (Gleason score: 3+4=7, ISUP group 2) with local visible neurological invasion (HE staining, 200×). (B) At two months after radical prostatectomy, the three-dimensional reconstruction of the urinary plain CT scan showed no recurrence of tumors. CT: Computed tomography; HE: Hematoxylin-Eosin; HP: Histopathological; ISUP: International Society of Urological Pathologists.

The patient has been in periodic follow-up and has remained in good general condition without any evidence of recurrence to date. The patient signed informed consent forms, allowing for publication of the relevant clinical and imaging data from his case.

\section{ㅁ Discussions}

Several reports have noted that the incidence of $\mathrm{PCa}$ in RCC patients is greater than the anticipated incidence rates in the general population. In a population study based on calculated standardized incidence ratio (SIR, observed/expected), Barocas et al. [4] found that the incidence of $\mathrm{PCa}$ is significantly higher in men with RCC (SIR, 1.42), but not in men with lung cancer (SIR, 1.14), colon cancer (SIR, 1.10), or NHL (SIR, 0.97) [4]. In contrast, the incidence of RCC was not significantly higher in men with $\mathrm{PCa}$ (SIR, 1.25) than in men with lung cancer (SIR, 2.08), colon cancer (SIR, 1.37), or NHL (SIR, 1.7). The researchers speculated that although some common etiological factors are possible, it is also possible that RCC patients followed by a urologist might be screened more rigorously for $\mathrm{PCa}$ than patients with other primary malignancies. In another cohort study in Norway, researchers found that $16 \%$ of new RCC cases had at least one biopsy-verified primary malignant tumor other than RCC, and PCa was the most common concurrent tumor [5]. However, when the other primary malignancies were divided into antecedent, synchronous, or subsequent (synchronous tumors were defined as other primary malignancies diagnosed $<91$ days before or after RCC diagnosis) in the 30 total concurrent PCa cases, only two were found to be synchronous. Joung et al. [6] also found that in Korea, for patients with primary RCC, the risk of developing a second primary cancer was higher than the risk of developing cancer in the general population (SIR, 1.13; 95\% confidence interval, 1.08 to 1.18 ). RCC survivors also have been observed to have elevated risk of PCa (SIR, 1.76). In an early study on the data available in the Surveillance Epidemiology and End Results database, researchers observed that about $10 \%$ of all patients with RCC had one or more new primary tumors [7]. The SIR of second primary cancers in RCC was 1.18 , which was significantly greater than expected. Solid tumors comprised $90 \%$ of all second malignancies in RCC patients, with the most common second cancers reported in the prostate gland and the digestive and respiratory systems. As shown in Table 1, in recent years, only five synchronous primary PRAD cases have been reported in RCC patients, with an average age of 68 [8-12]. Most cases (three cases) were diagnosed as RCC of Furhman grade II and PRAD of Gleason score more than 6 . Moreover, more $50 \%$ of cases had symptoms. It should also be noted that elevated total PSA levels were only detected in two cases $[10,12]$.

Table 1 - Summary of case reports of synchronous PRAD in RCC patients

\begin{tabular}{|c|c|c|c|c|c|c|c|}
\hline Case report & $\begin{array}{c}\text { Patient age } \\
\text { [years] }\end{array}$ & Primary cancers & Symptoms & $\begin{array}{c}\text { RCC Fuhrman } \\
\text { grade }\end{array}$ & $\begin{array}{c}\text { tPSA [ng/mL] } \\
\text { (fPSA/tPSA) }\end{array}$ & $\begin{array}{c}\text { PRAD Gleason } \\
\text { score }\end{array}$ & IHC \\
\hline Present & 62 & RCC, PRAD & Renal mass & II & $6.03(0.12)$ & $6(3+3)$ & In text \\
\hline Elec et al. [8] & 78 & RCC, PRCC, PRAD, SCCB & Uremia & I & $1.41(\mathrm{ND})$ & $6(3+3)$ & ND \\
\hline Pastore et al. [9] & 70 & RCC, PRAD, OPSCC & LUTS & II & 3.91 (ND) & $8(4+4)$ & ND \\
\hline $\begin{array}{l}\text { Zargar-Shoshtari } \\
\text { et al. [10] }\end{array}$ & 71 & RCC, PRAD, male IDC & None & II & 7.90 (ND) & $7(3+4)$ & ND \\
\hline Coyle et al. [11] & 69 & RCC, PRAD, LPS & ND & II & ND & ND & ND \\
\hline Tiwari et al. [12] & 55 & RCC, PRAD, TCCB & Uremia & ND & 12.00 (ND) & ND & ND \\
\hline
\end{tabular}

fPSA/tPSA: Free/total prostate-specific antigen ratio; IDC: Invasive ductal carcinoma; IHC: Immunohistochemistry; LPS: Liposarcoma; LUTS: Lower urinary tract symptoms; ND: No description provided; OPSCC: Oropharyngeal squamous cell carcinoma; PRAD: Prostate adenocarcinoma; PRCC: Papillary renal cell carcinoma; RCC: Renal cell carcinoma; SCCB: Small cell carcinoma of the bladder; TCCB: Transitional cell carcinoma of bladder. 
However, some important information, such as fPSA/tPSA ratio and tumor IHC results has not been provided in these cases. Therefore, to the best of our knowledge, the present report is the only case with systematically described pre- and post-therapy laboratory, histology, IHC, and imaging examination results.

Different cancers synchronously found in the same patient are referred to as multiple primary malignancies [13]. Warren \& Gates [14] described the criteria for identifying multiple primary malignancies in the 1930s as: each tumor must have a definite malignancy and distinct histological characteristics; and metastasis of another tumor must be excluded. For modern oncology, tumors are considered multiple primaries only if they are detected at different locations by imaging examination and have different microscopic and/or molecular pathology patterns [13]. A differential diagnosis should be created according to these criteria between multicenter tumors or metastases and multiple primaries. In this study, RCC was initially found by CT scan in the patient with complaint of a painless renal mass, whereas $\mathrm{PCa}$ was incidentally considered by abnormal PSA level results. MRI revealed uneven signals in the prostate right peripheral zone. A postoperative pathology assay reported clear RCC with positive staining of vimentin, CD10, CA-IX, Pax-8, EMA, and $\mathrm{Ki} 67 \mathrm{LI}$, as well as prostate acinar adenocarcinoma with positive staining of P504S and Ki67 LI. Taken together, these pieces of evidence strongly supported our diagnosis of concurrence primary RCC and $\mathrm{PCa}$ other than metastasis and multifocal tumors.

Although some common genetic and/or environmental risk factors are believed to exist, the exact underlying common pathogenesis of concurrence primary RCC and $\mathrm{PCa}$ has yet to be explained [15]. On the other hand, approximately $75 \%$ of RCC cases have been found in individuals over 60 years old and the incidence increases with age [15]. Therefore, with increased longevity and improved survival due to novel treatments, patients with RCC are more likely to develop extra malignancies, such as PCa. Thus, strict monitoring would help us for the early detection of synchronous or subsequent PCa. Although PSA tests combined with biopsy are effective for screening PCa, up to $20 \%$ of patients with negative biopsy results have subsequently been found to have cancer. Therefore, increasing use of CT scanning and MRI for primary evaluation should be helpful. In addition, as we have done for this patient, the most aggressive tumor should be treated as a priority. After improving the overall general condition of the patient, other malignancies could be properly managed.

\section{무 Conclusions}

Our report together with published studies suggest that increased awareness of the risk of simultaneous primary $\mathrm{PCa}$ will enable early detection and prompt therapies in patients with RCC.

\section{Competing interests}

The authors declare that they have no competing interests.

\section{Funding}

No funding was received.

\section{Acknowledgments}

Thanks to the help of Yu-Xiang Shi, MD, PhD, from the Department of Pathology in our Hospital.

\section{Availability of data and materials}

The datasets analyzed during the current study are available from the corresponding author on reasonable request.

\section{Authors' contribution}

ZYL and CCY analyzed and interpreted the patient data regarding the imaging examination and the pathology. ZHW and ZSW performed the pictures of computed tomography. GHL and LC were contributors in writing the manuscript. YLG commented in detail on the drafts. All authors read and approved the final manuscript.

Zhong-Yuan Li and Cheng-Cheng Ying contributed equally to this work.

\section{Ethics approval and consent to participate}

The present study was approved by the Ethics Committee of The Central Hospital of Wuhan, Tongji Medical College, Huazhong University of Science and Technology. All patients provided written informed consent prior to the study start.

\section{Patient consent for publication}

The patient signed informed consent forms, allowing for publication of the relevant clinical and imaging data from his case.

\section{References}

[1] Bray F, Ferlay J, Soerjomataram I, Siegel RL, Torre LA, Jemal A. Global cancer statistics 2018: GLOBOCAN estimates of incidence and mortality worldwide for 36 cancers in 185 countries. CA Cancer J Clin, 2018, 68(6):394-424. https:// doi.org/10.3322/caac.21492 PMID: 30207593

[2] Capitanio U, Bensalah K, Bex A, Boorjian SA, Bray F, Coleman J, Gore JL, Sun M, Wood C, Russo P. Epidemiology of renal cell carcinoma. Eur Urol, 2019, 75(1):74-84. https:// doi.org/10.1016/j.eururo.2018.08.036 PMID: 30243799

[3] Rabbani F, Grimaldi G, Russo P. Multiple primary malignancies in renal cell carcinoma. J Urol, 1998, 160(4):1255-1259. PMID: 9751330

[4] Barocas DA, Rabbani F, Scherr DS, Vaughan ED Jr. A population-based study of renal cell carcinoma and prostate cancer in the same patients. BJU Int, 2006, 97(1):33-36. https://doi.org/10.1111/j.1464-410X.2005.05880.x PMID: 16336324

[5] Beisland C, Talleraas O, Bakke A, Norstein J. Multiple primary malignancies in patients with renal cell carcinoma: a national population-based cohort study. BJU Int, 2006, 97(4):698702. https://doi.org/10.1111/j.1464-410X.2006.06004.x PMID: 16536756

[6] Joung JY, Kwon WA, Lim J, Oh CM, Jung KW, Kim SH, Seo HK, Park WS, Chung J, Lee KH, Won YJ. Second primary cancer risk among kidney cancer patients in Korea: a population-based cohort study. Cancer Res Treat, 2018, 50(1):293-301. https://doi.org/10.4143/crt.2016.543 PMID: 28421722 PMCID: PMC5784635

[7] Chakraborty S, Tarantolo SR, Batra SK, Hauke RJ. Incidence and prognostic significance of second primary cancers in renal cell carcinoma. Am J Clin Oncol, 2013, 36(2):132-142. https:// doi.org/10.1097/COC.0b013e3182438ddf PMID: 22441339 PMCID: PMC3383896

[8] Elec FI, Zaharie A, Ene BM, Ghervan L. Quadruple primary urogenital cancers - a case report. Int J Surg Case Rep, 2017, 39:239-244. https://doi.org/10.1016/j.ijscr.2017.08.035 PMID: 28858743 PMCID: PMC5581378 
[9] Pastore AL, Palleschi G, Leto A, Silvestri L, Porta N, Petrozza V, Carbone A. A novel combination of triple metachronous malignancies of the kidney, oropharynx and prostate: a case report. Oncol Lett, 2015, 10(2):917-920. https://doi.org/10.3892/ol.2015.3312 PMID: 26622595 PMCID: PMC4509434

[10] Zargar-Shoshtari MA, Saffari H, Moslemi MK. Metachronous occurrence of triple malignancies of kidneys, prostate, and breast. A case report and review of the literature. Case Rep Urol, 2013, 2013:194620. https://doi.org/10.1155/2013/194620 PMID: 23533925 PMCID: PMC3606744

[11] Coyle D, Flaherty RA, Kelly BD, Hynes SO, Colesky F Quinlan MR, Corcoran MO, Rogers E. Recurrent dedifferentiated paratesticular liposarcoma with synchronous renal cell carcinoma and prostate cancer. Curr Urol, 2013, 6(4):216-218. https://doi.org/10.1159/000343543 PMID: 24917747 PMCID: PMC3783303
[12] Tiwari P, Tripathi A, Bansal P, Vijay M, Gupta A, Kundu AK. Synchronous primary cancers of urinary bladder and kidney and prostate. Saudi J Kidney Dis Transpl, 2012, 23(4): 786-789. https://doi.org/10.4103/1319-2442.98161 PMID: 22805392

[13] Vogt A, Schmid S, Heinimann K, Frick H, Herrmann C, Cerny T, Omlin A. Multiple primary tumours: challenges and approaches, a review. ESMO Open, 2017, 2(2):e000172. https://doi.org/ 10.1136/esmoopen-2017-000172 PMID: 28761745 PMCID: PMC5519797

[14] Warren S, Gates O. Multiple primary malignant tumors: a survey of the literature and statistical study. Am J Cancer, 1932, 16:1358-1414.

[15] Qayyum T, Oades G, Horgan P, Aitchison M, Edwards J. The epidemiology and risk factors for renal cancer. Curr Urol, 2013, 6(4):169-174. https://doi.org/10.1159/000343534 PMID: 24917738 PMCID: PMC3783274

\section{Corresponding author}

Yong-Lian Guo, MD, PhD, Director of the Urology, Department of Urology, The Central Hospital of Wuhan, Tongji Medical College, Huazhong University of Science and Technology, 26 Shengli Street, Jiangan District, 430014 Wuhan, People Republic of China; Phone +86 278221 1667, e-mail: 410480215@qq.com

Received: March 14, 2020

Accepted: October 2, 2020 